\title{
Klasse-IV-Evidenz für Rituximab
}

Fragestellung: Ist Rituximab in der Therapie der Anti-MuSKantikörpervermittelten Myasthenia gravis wirksam?

Hintergrund: Die Anti-MuSK-antikörpervermittelte Form der Myasthenia gravis (MG) ist innerhalb der Erkrankungen der neuromuskulären Endplatte selten. Die Erkrankung zeichnet sich häufig durch einen schweren okulobulbär betonten Verteilungstyp aus. Häufig lässt sich unter der konventionellen immunsuppressiven Behandlung keine vollständige Remission der neuromuskulären Beschwerden erreichen, sodass die Therapie eine besondere Herausforderung bedeutet. Rituximab hat sich in mehreren Fallsammlungen und kleineren Open-labelStudien als möglicherweise wirksam herausgestellt. Kontrollierte Studien für diese Fragestellung fehlen sowohl in der Behandlung der Acetylcholinrezeptor-antikörperpositiven MG als auch der Verlaufsform, die durch Anti-MuSK-Antikörper vermittelt wird. In diesem multizentrischen Ansatz wurde nun in Form eines verblindeten prospektiven Reviewprozesses die Wirksamkeit von Rituximab in der Behandlung der Anti-MuSK-antikörpervermittelten MG untersucht.

Patienten und Methodik: 77 von 119 Patienten mit einer AntiMuSK-antikörpervermittelten MG wurden im Zeitraum 2005 bis 2015 an zehn neuromus-

Hehir MK, Hobson-Web LD, Benatar $\mathrm{M}$ et al. Rituximab as treatment for anti-MuSK myasthenia gravis. Multicenter blinded prospective review. Neurology 2017; 89: 1069-77 kulären Zentren in Form eines besonderen Reviewprozesses analysiert. Die Besonderheit der Studie ist das retrospektive Studiendesign mit einem verblindeten prospektiven Reviewprozess, da dop- pelblind randomisierte, kontrollierte Studien aufgrund der geringen Patientenzahl nicht sicher durchführbar sind. Die Behandlungsdaten wurden jeweils fünf verblindeten Prüfern vorgelegt, die den Schweregrad, das immunsuppressive Behandlungsregime sowie den Verlaufstyp der Erkrankung einordnen sollten. Ein Einschluss in die Studie erfolgte, wenn mindestens vier von fünf Prüfern der Teilnahme an der Studie anhand der vorher festgelegten Einschlusskriterien zugestimmt hatten. Von 119 gescreenten Patienten wurden letztlich 77 in die Studie eingeschlossen. 22 Patienten wurden aufgrund eines nicht ausreichenden Follow-up im Verlauf ausgeschlossen. 24 Patienten erhielten Rituximab, 31 Patienten wurden als Kontrollpatienten definiert. Die mediane Follow-up-Dauer war länger als 3,5 Jahre. Der primäre Outcomeparameter war die Kombination aus dem klinischen Schweregrad (MGFA) sowie dem MGFA-postinterventionellen Status (PIS), der Anzahl der Immunsuppressiva sowie deren jeweiliger Dosis. Der primäre Endpunkt wurde erreicht wenn der kombinierte MGFA + PIS Score (MGSTI) $\leq 2$ betrug.

Ergebnisse: In der Rituximab-Gruppe erreichten $58 \%$ der Patienten den primären Endpunkt, verglichen mit $16 \%$ in der Kontrollgruppe $(p=0,002)$. Die Number needed to treat des primären Outcomeparameters wurde mit 2,4 errechnet. Bei Studienende wurden noch $29 \%$ der mit Rituximab behandelten Patienten mit Prednison behandelt versus $74 \%$ der Patienten in der Kontrollgruppe.

Schlussfolgerungen: Die Autoren geben eine Klasse-IV-Evidenz für die Wirksamkeit von Rituximab in der Behandlung der Anti-MuSK-antikörpervermittelten MG.

\section{- Kommentar von Tim Hagenacker, Essen}

\section{Geschicktes Studiendesign zur Untersuchung seltener Erkrankungen}

Die Ergebnisse bei dieser langen Studiendauer zeigen erneut die Wirksamkeit von Rituximab in der Behandlung der AntiMuSK-antikörpervermittelten MG. Diese Patienten sind häufig therapierefraktär, sodass sie langfristig mit hohen Kortikosteroiddosen und weiterhin hohen neuromuskulären Einschränkungen und den daraus folgenden Konsequenzen zu kämpfen haben. Aufgrund der Seltenheit der Anti-MuSK-antikörpervermittelten MG sind randomisierte kontrollierte Doppelblindstudien zu diesem Thema häufig nicht umsetzbar beziehungsweise bereits in der Planung zum Scheitern verurteilt, sodass die Autoren hier mit einem innovativen Studiendesign und einer Kombination aus retrospektiver Datenerhebung und prospektiver Analyse versuchen, dieses Problem zu umgehen. Die Schwächen der Studie zeigen sich zum einen im primären Endpunkt, da der hier verwendete MGSTI-Score mit einer Kombination aus MGFA + PIS bisher nicht für diese Erkrankung validiert wurde. Zudem lässt die lange Rekrutierungsdauer von rund zehn Jahren durch die möglicherweise wechselnden Untersucher einen Bias in der Datenerhebung vermuten. Dennoch handelt es sich um eine wichtige Studie, da diese erneut die Wirksamkeit von Rituximab in dieser seltenen Patientengruppe zeigt und somit im häufigen Problemfeld der Kostenübernahme der Therapie die Datenlage verbessert.

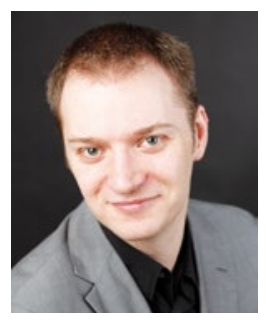

PD Dr. med. Tim Hagenacker, Essen

Klinik für Neurologie,

Universitätsklinikum Essen

E-Mail: tim.hagenacker@uk-essen.de 